\title{
Underwater holmium-laser-pulse-induced complete cavitation bubble movements and acoustic transients
}

\author{
LÜ Tao ${ }^{1,2}$ \& LI ZhengJia ${ }^{1 *}$ \\ ${ }^{1}$ Wuhan National Laboratory for Optoelectronics, Huazhong University of Science and Technology, Wuhan 430074, China: \\ ${ }^{2}$ School of Mathematics and Physics, China University of Geosciences, Wuhan 430074, China
}

Received September 29, 2010; accepted December 6, 2010

\begin{abstract}
The complete evolution of holmium-laser-pulse-induced cavitation bubble movements and acoustic transients underwater are investigated experimentally. The laser was single fiber-guided and had a $300 \mathrm{~mJ}$ pulse energy and $300 \mu$ s pulse duration (full width at half-maximum). In our experiments, more than four oscillations and four acoustic transients were demonstrated. $272 \mu \mathrm{s}$ after laser onset, the cavitation bubbles reached their maximum transverse and longitudinal lengths of 2046 and $1914 \mu \mathrm{m}$, respectively. The maximum transverse and longitudinal bubble wall velocities were 28.9 and $39.2 \mathrm{~m} / \mathrm{s}$ at 560 and $528 \mu$ s after laser onset, respectively. This investigation will be helpful to make good use of cavitation effect in medical applications of holmium laser pulses.
\end{abstract}

cavitation bubble, acoustic transient, holmium laser pulse

Citation: $\quad$ Lü T, Li Z J. Underwater holmium-laser-pulse-induced complete cavitation bubble movements and acoustic transients. Chinese Sci Bull, 2011, 56: 1226-1229, doi: 10.1007/s11434-011-4367-5

Pulsed holmium lasers at wavelengths of $2.12 \mu \mathrm{m}$ have attracted considerable interest, because of their applications in the precise removal of biological tissues [1]. When the optical fiber is not in direct contact with the target tissue, cavitation bubbles (CB) are formed by absorption of the laser radiation by the liquid separating the fiber tip and the tissue surface [2]. These bubbles can directly affect the laser ablation process [3]. After formation, the $\mathrm{CB}$ undergoes multiple cycles of expansion and collapse, accompanying shockwave emission [2,4]. However, many studies have reported that this evolution is not sequential but transient. All images must be reconstructed from different $\mathrm{CBs}$ induced by pulsed lasers with identical parameters [5] by performing shadow and Schlieren photography [2,4-10]. However, because of the poor pulse-to-pulse repeatability of these laser sources, the pulse width, pulse energy and response time always fluctuate even under the same ambient environments. Consequently, the presented images cannot

*Corresponding author (email: Zhjli@mail.hust.edu.cn) represent the true dynamics of the CBs [6]. Therefore, it is necessary to visualize the complete evolution of a $\mathrm{CB}$ induced by a single holmium laser pulse. This will aid in the explanation of the full effect of CBs on tissue ablation applications.

A schematic diagram of the experimental setup is shown in Figure 1. A DG645 digital delay/pulse generator (Stanford Research Systems, USA) was linked to a holmium laser system (Wuhan National Laboratory for Optoelectronics), a high-speed camera (FASTCAM SA1.1, Japan) and an oscillograph (Agilent Technologies InfiniVision, 350 $\mathrm{MHz}, 2 \mathrm{GSa} / \mathrm{s}$ ). After the LED light source (Blog, SLT-P007, LED luminance $>12000$ Mcd) was initiated independently, the pulse generator could be used in a single-shot-triggering mode. The bubble dynamics and acoustic transients were recorded and detected using the camera and needle hydrophone (Institute of Acoustics, Chinese Academy of Sciences, sensitivity $>10 \mathrm{nV} / \mathrm{Pa}$ ), respectively. The distal fiber tip was placed in a $10 \mathrm{~cm} \times 10 \mathrm{~cm} \times 10 \mathrm{~cm}$ quartz container filled with pure water, and was kept $3 \mathrm{~cm}$ 


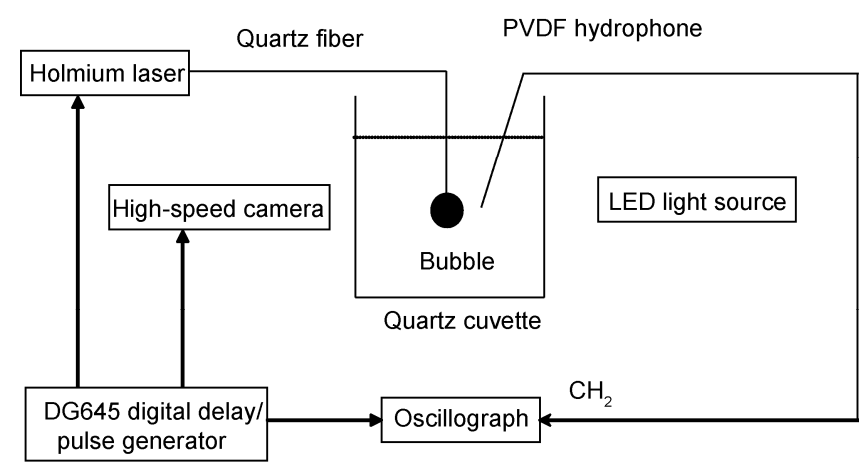

Figure 1 Schematic diagram of the experimental setup.

below the water surface to insure sufficient inertial confinement.

It can be seen from Figure 2 that more than four oscillations occurred. Brightness variations could be observed for more than three oscillations. However, the corresponding volume variations could not be confirmed. Less than $48 \mu \mathrm{s}$ after laser onset, the $\mathrm{CB}$ began to form, which agrees well with previously reported results [8]. The CB was pearshaped during the first oscillation period, but the CB separated from the fiber tip at the beginning of the second oscillation period and took on a mushroom-shape. At the beginning of the third period, the bubble shape was more irregular. At $616 \mu$ s, the CB collapsed and then divided into two parts. During the fourth oscillation period at $632 \mu \mathrm{s}$, the two parts then recombined into one that had an increased diameter $495 \mu \mathrm{m}$. After $632 \mu \mathrm{s}$, the diameter of the bubble stayed approximately constant at $495 \mu \mathrm{m}$.

In Figure 3 the maximal transverse lengths can reach 2046, 1036, 693, $495 \mu \mathrm{m}$ at 272, 484, 600, $632 \mu$ s after laser onset, respectively. The maximal longitudinal lengths can reach $1914,1386,660,495 \mu \mathrm{m}$ at $272,512,592,632 \mu \mathrm{s}$ after laser onset, respectively. From 64 to $224 \mu \mathrm{s}$, the corre- lation of the transverse and longitudinal diameters of CBs with respect to time are approximately linear. Their transverse and longitudinal dimeters vary from 660 and $495 \mu \mathrm{m}$ to 1980 and $1815 \mu \mathrm{m}$, respectively. Then they maintain the same diameters of 1980 and $1815 \mu \mathrm{m}$ from 224 to $240 \mu \mathrm{s}$. At $256 \mu$ s they increase to 2046 and $1914 \mu \mathrm{m}$, respectively, and maintain the same diameters for about $32 \mu$ s (from 256 to $288 \mu \mathrm{s})$. Another important phenomenon during every oscillation period, especially for the first, is that the slopes of the diameter change with respect to time for transverse diameters are slower than those of longitudinal diameters. The last important phenomenon is that the transverse diameters are always bigger than the longitudinal diameters, except when close to the maximum expansion phase during the second expansion phase because of a small part of bubble attached to the fiber tip (this phenomenon vanishes after $484 \mu \mathrm{s})$. In Figure 4 during the entire oscillation process the longitudinal bubble wall velocities are always faster than the transverse bubble wall velocities, and their maximal velocities reach 39.2 and $28.9 \mathrm{~m} / \mathrm{s}$ at 528 and $560 \mu$ s after laser onset, respectively.

To avoid the acoustic transients induced by bubble collapse, which can damage the hydrophone, and effects from the hydrophone on the bubble dynamics, the distance between the tips of the hydrophone and the fiber was set to 4 $\mathrm{mm}$. The absolute pressure measurement results, without the preamplifier, are shown in Figure 5. In addition to the strong pressure peaks (marked 1-4), which were induced by the collapse phases of the bubble oscillations (1-4), there is a slow increase in pressure (shown by the arrow) after the beginning of the laser pulse. This is an indication of $\mathrm{CB}$ formation [6]. The pressures were 23.375, 8, 6.7 and 2.96 bar for peaks 1, 2, 3 and 4, respectively. The minimum bubble radius is smaller than $300 \mu \mathrm{m}$ (see image taken at 416 $\mu$ s in Figure 2). Therefore, if we assume a $1 / r$ damping of
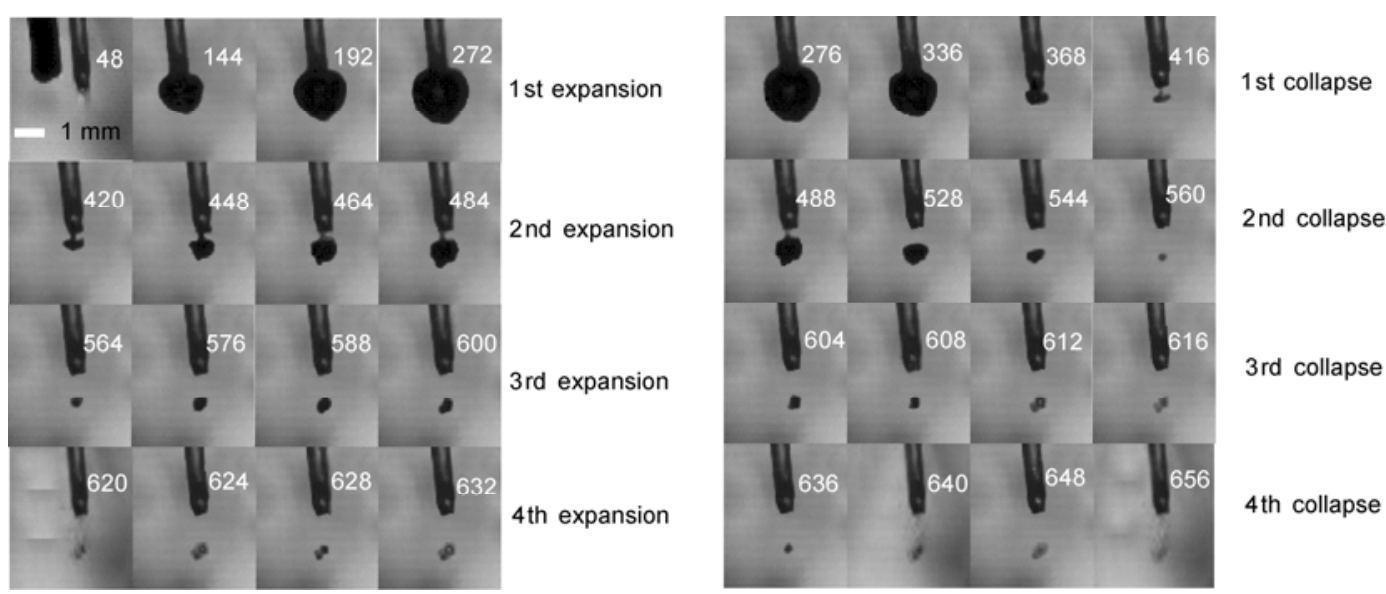

Figure 2 Sequential images of CB formation recorded using a high-speed camera with a frame rate of 25000 frames per second. The time ( $\mu$ s) given for each image represents the time after the onset of a single holmium laser pulse $\left(E_{\mathrm{p}}=300 \mathrm{~mJ}\right.$, duration $\left.=300 \mu \mathrm{s}\right)$. The pulses were delivered via a fiber of 600 $\mu \mathrm{m}$ core diameter and a $660 \mu \mathrm{m}$ cladding diameter. The object on the left side of the first picture is the needle hydrophone. 


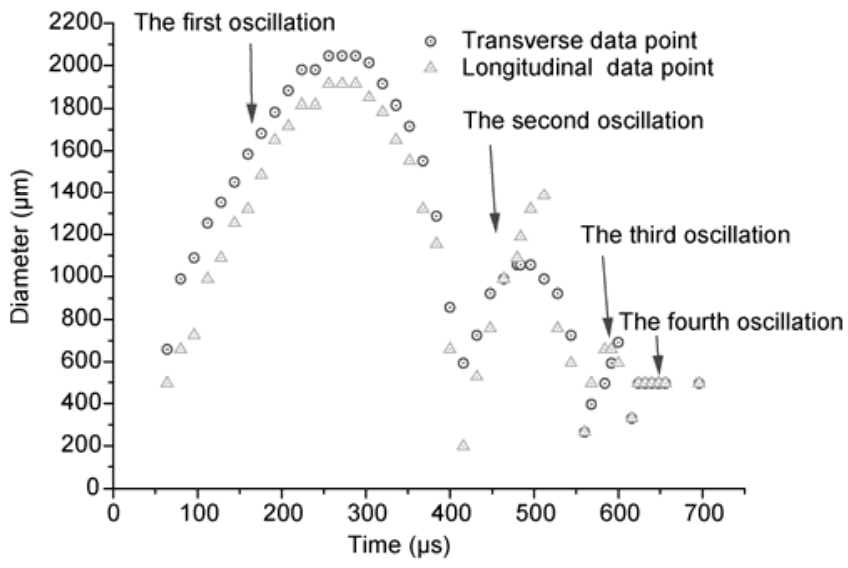

Figure 3 Time evolution of the transverse and longitudinal lengths.

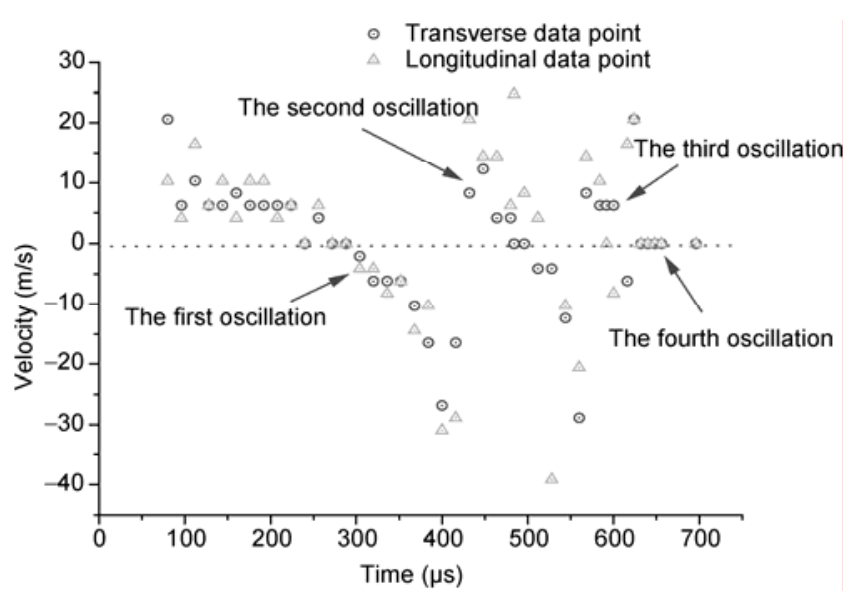

Figure 4 Time evolution of the transverse and longitudinal velocities of the bubble walls. $V>0$ and $V<0$ denote expansion and collapse velocities, respectively.

the pressure, where $r$ is the distance from the collapse center, we calculate a pressure of more than 312 bar at a distance of $300 \mu \mathrm{m}$ from the collapse center. From the highspeed photography results (Figure 2), it can be seen that 40 $\mu$ s after holmium laser onset the $\mathrm{CB}$ begins to form. Because the time interval between the bubble formation and the first acoustic transient is $278 \mu$ s, we calculated that the first pressure pulse is generated $18 \mu$ s after the end of holmium laser pulse. This agrees with the findings in [6].

Assuming that the temporal profiles of the positive pressure waves (shown in Figure 5 I, II, III and IV) can be approximated by exponential pulses with full width at half maximum pulse durations equal to those given by the hydrophone measurements [11], we calculated the relevant parameters of the corresponding four shock waves. These are listed in Table 1. The efficiency of energy conversion from the holmium laser pulse to acoustic transients was up to $0.0927 \%$.

In our experimental results, the four shock waves had bipolar shapes with a positive compression wave followed by a negative wave. However, other researchers [2,5-10]

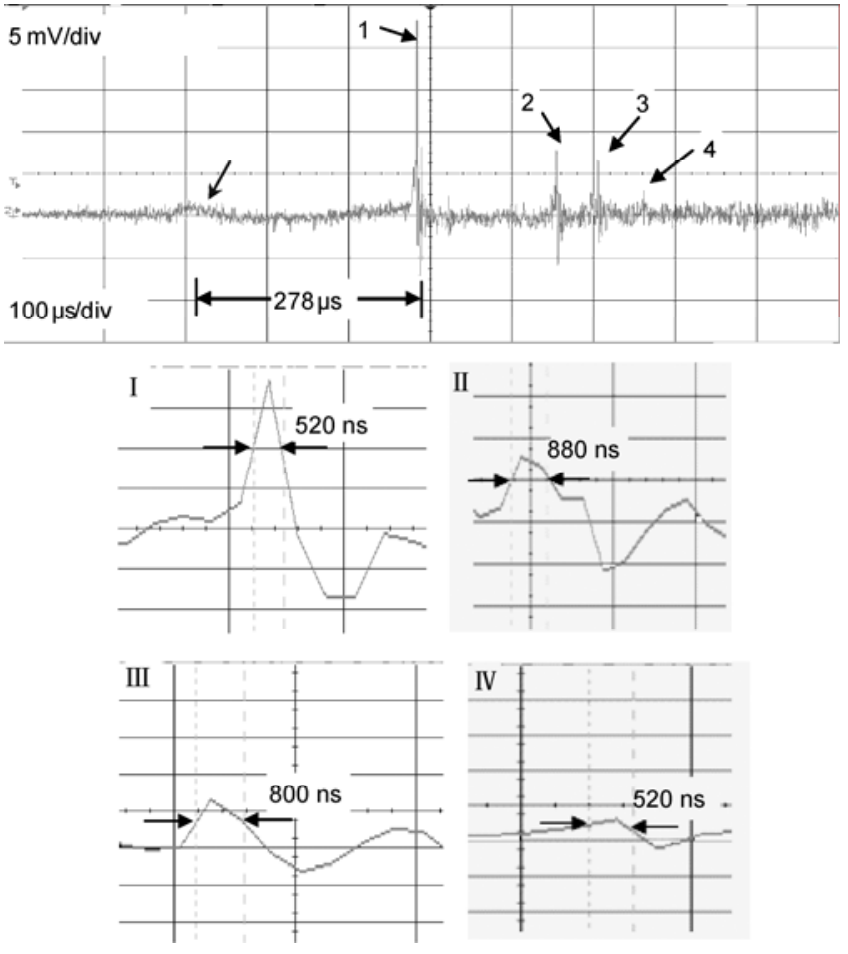

Figure 5 Oscilloscope traces of the acoustic transients induced by the CB evolution $\left(E_{\mathrm{p}}=300 \mathrm{~mJ}\right.$, duration $\left.=300 \mu \mathrm{s}\right)$ at the submerged fiber tip $(600$ $\mu \mathrm{m})$. The four shock waves, 1, 2, 3 and 4 are shown on shorter time scales in I, II, III and IV, respectively.

Table 1 Parameters of the acoustic transients

\begin{tabular}{ccccc}
\hline Acoustic transient & $\Delta t_{\mathrm{s}}(\mathrm{ns})$ & $p_{\mathrm{s}}(\mathrm{MPa})^{\mathrm{a})}$ & $E_{\mathrm{s}}(\mu \mathrm{J})^{\mathrm{b})}$ & $E_{\mathrm{s}} / E_{\mathrm{p}}(\%)$ \\
\hline No. 1 & 520 & 2.3375 & 278 & 0.0927 \\
No. 2 & 880 & 0.8023 & 55.5023 & 0.0183 \\
No. 3 & 800 & 0.6744 & 36.6517 & 0.0122 \\
No. 4 & 520 & 0.2965 & 4.437 & 0.0015 \\
\hline
\end{tabular}

a) $p_{\mathrm{s}}$, Shock wave peak pressure; b) $E_{\mathrm{s}}$, shock wave energy.

reported there was only a positive compression wave, which are induced by free-running holmium lasers (pulse widths of several hundreds $\mu \mathrm{s})$. However, our observation can be explained in terms of momentum conservation. If the cavitation threshold is exceeded, the total momentum, which is proportional to the time integral over the stress wave, must remain zero. On the other hand, the initial asymmetry of the bipolar wave must be compensated by this additional negative stress [12]. Furthermore, the response time (several decades nanoseconds) of the needle hydrophone is too slow to satisfy the experimental requirements. Therefore, this phenomenon must be further investigated theoretically and experimentally in future work.

In conclusion, the complete evolution of holmium-laserpulse-induced cavitation bubble movements and acoustic transients was investigated. An acoustic transient with peak compress wave of 312 bar was generated by the first collapse of the induced $\mathrm{CB}$. This suggests that $\mathrm{CB}$ formation is a potential cause of acoustical tissue damage during medical 
application of holmium laser pulses in a liquid environment such as arthroscopic or angioplasty surgery.

This work was supported by the National Natural Science Foundation of China (61008054) and the Fundamental Research Funds for the Central Universities, China University of Geosciences (Wuhan) (CUG090112).

1 Vogel A, Venugopalan V. Chem Rev, 2003, 103: 577-644

2 Frenz M, Paltauf G, Schmidt-kloiber H. Phys Rev Lett, 1996, 76: 3546-3549

3 Wagner W, Sokolow A, Peartstein R, et al. Appl Phys Lett, 2009, 94 : 013901
4 Brinkmann R, Hansen C, Mohrenstecher D, et al. IEEE J Quant Electron, 1996, 2: 826-835

5 Asshauer T, Rink K, Delacretaz G. J Appl Phys, 1994, 76: 50075013

6 Frenz M, Konz F, Pratisto H, et al. J Appl Phys, 1998, 84: 5905-5912

7 Frenz M, Pratisto H, Konz F, et al. IEEE J Quant Electron, 1996, 32: 2025-2035

8 Asshauer T, Delacretaz G, Jansen E D, et al. Appl Phys B, 1997, 65: 647-657

9 Jansen E D, Asshauer T, Frenz M, et al. Lasers Surg Med, 1996, 18: 278-293

10 Kang H W, Lee H, Teichman J M H, et al. Lasers Surg Med, 2006, 38: 762-772

11 Vogel A, Noack J, Nahen K, et al. Appl Phys B, 1999, 68: 271-280

12 Paltauf G, Kloiber H S. Appl Phys A, 1996, 62: 303-311

Open Access This article is distributed under the terms of the Creative Commons Attribution License which permits any use, distribution, and reproduction in any medium, provided the original author(s) and source are credited. 